\title{
Landau levels in asymmetric graphene trilayers
}

\author{
S. H. R. Sena, ${ }^{1}$ J. M. Pereira Jr., ${ }^{1}$ F. M. Peeters,,${ }^{1,2}$ and G. A. Farias ${ }^{1}$ \\ ${ }^{1}$ Departamento de Física, Universidade Federal do Ceará, Fortaleza, Ceará, 60455-760, Brazil \\ ${ }^{2}$ Department of Physics, University of Antwerp, Groenenborgerlaan 171, B-2020 Antwerpen, Belgium \\ (Received 1 July 2011; revised manuscript received 19 October 2011; published 21 November 2011)
}

\begin{abstract}
The electronic spectrum of three coupled graphene layers (graphene trilayers) is investigated in the presence of an external magnetic field. We obtain analytical expressions for the Landau level spectrum for both the $A B A$ and $A B C$ type of stacking, which exhibit very different dependence on the magnetic field. We show that layer asymmetry and an external gate voltage can strongly influence the properties of the system.
\end{abstract}

DOI: 10.1103/PhysRevB.84.205448

PACS number(s): 71.10.-w, 73.21.-b, 73.22.Pr

\section{INTRODUCTION}

The extraordinary level of interest in the study of single layer graphene has led to the prediction and observation of several unusual phenomena not found in other low-dimensional systems. These new properties are mainly a consequence of the chiral and massless character of the quasiparticles in graphene. ${ }^{1}$ This new material is not only expected to lead to several technological applications, but has also helped shed light on relativistic quantum effects, such as Klein tunneling ${ }^{2,3}$ and zitterbewegung (see, e.g., Refs. 4 and 5). There is currently a search for experimental methods for producing high quality samples of graphene in large quantities. However, the current experimental techniques can also create carbon structures with two or more layers. It has already been recognized that graphene bilayers (i.e., two coupled layers of graphene) can display interesting new properties that are distinct from those of single layers ${ }^{6-8}$ and that can also be eventually harnessed for the development of devices. One important aspect of these structures is the fact that, in comparison with the in-plane interactions, the comparatively weaker interlayer coupling can yet exert a significant influence on the carrier spectrum. Thus, whereas the electronic dispersion at the vicinity of the Dirac point in single layer graphene is linear, in bilayer graphene it displays an approximately parabolic shape with the appearance of higher energy bands. Moreover, in striking contrast with single layers, the electronic spectrum of bilayer graphene has been shown to develop a gap in the presence of an external electric field. Thus the interlayer coupling in stacked layers of graphene gives rise to a rich set of properties that are not found in monolayers, and can be expected to be of particular significance in structures with three or more layers. In particular, it has been shown that, in the nearest-neighbor coupling approximation, for a stack of $N$ layers of graphene, there should exist $N$ distinct Landau levels at $E=0$ for each spin and valley. ${ }^{9}$

In this work we investigate the properties of three coupled layers of graphene, i.e., trilayer graphene (TLG) in the presence of an external magnetic field perpendicular to the plane of the layers. The properties of TLG in the absence of a magnetic field have been considered in the literature within a tight-binding model (see, e.g., Refs. 10 and 11), as well as through first-principles calculations. ${ }^{12}$ The effect of an external magnetic field was calculated by means of an approximation based on the mapping of stacked graphene layers to a onedimensional (1D) tight-binding chain by Guinea et al. ${ }^{13}$ The effect of disorder on the transport properties of TLG has also been studied theoretically. ${ }^{14,15}$ Recent experimental ${ }^{16-18}$ and theoretical ${ }^{19-21}$ studies have investigated the Landau-level spectrum of unbiased TLG and recent magnetoconductance measurements of TLG have been performed. ${ }^{22-25}$ These results showed that one important aspect of TLG is the fact that the energy bands at the vicinity of the Dirac point are very sensitive to the particular type of stacking of the layers. The two more relevant stacking rules are the rhombohedral, or $A B C$ stacking, and the Bernal, or $A B A$ stacking. In each case, the relative position of the lowermost layer ( $C$ in one case and $A$ in the other) helps dictate the possible symmetries of the subsequent wave functions associated with each layer. The type of stacking is also relevant to the properties of the Landau levels of the TLG, and it has recently been reported ${ }^{26}$ that $15 \%$ of exfoliated TLG has rhombohedral $(A B C)$ stacking. For the case of $A B A$-stacked TLG, Koshino et al. have shown that the Landau-level spectrum shows energy levels grouped in two bands: one of those bands displays a "monolayerlike" dependence on $\sqrt{B}$, whereas the other band has a linear, "bilayerlike" magnetic-field dependence. The goal of the present paper is to present analytical results for the spectrum of TLG in a magnetic field considering different potentials in each layer. In contrast with previous numerical work (see, e.g., Ref. 20), we perform a direct diagonalization of the six-band continuum model and obtain analytical expressions for the Landau-level spectrum as a function of magnetic field and the potentials at each layer, for both the $A B C$ and $A B A$ stackings. In particular, we calculate the TLG spectrum in the presence of electric fields that break the layer symmetry. It has recently been shown that these different potentials can lead to the opening of a gap in the TLG spectrum. ${ }^{11,27-29}$ In the present paper we focus on the modifications of the Landau levels as a function of the layer potential.

The paper is organized as follows: in Sec. II we present the model and solve the resulting system of equations for the $A B A$ stacking, followed by Sec. III in which we present the model and obtain solutions for the $A B C$ stacking. Section IV shows and discusses the calculated Landau-level spectra and finally, the results are summarized in the Conclusions.

\section{II. $A B$ A STACKING}

Let us consider a system consisting of three coupled graphene layers, in the context of the tight-binding model. We assume nearest-neighbor hopping between sites within each 
layer, described by the coupling parameter $t_{0}$. In the continuum approximation, this parameter determines the magnitude of the Fermi velocity $v_{F}=t_{0} a \hbar^{-1} \sqrt{3} / 2 \approx 10^{6} \mathrm{~m} / \mathrm{s}$. The nearestneighbor interlayer coupling scheme is $A_{1}-B_{2}-A_{3}$, with coupling parameter $t_{1}$ (see Fig. 1). Previous work ${ }^{20}$ has shown that the addition of second-nearest-neighbor coupling terms causes a small shift of the Dirac point and breaks the electron-hole symmetry. Furthermore, these couplings cause the appearance of trigonal warping. Since we are primarily focused on the low-energy range, where these effects are less relevant, we will neglect second-nearest-neighbor interactions between sites in adjacent layers. On the other hand, the second-nearest-neighbor coupling terms in the uppermost and lowermost layers were found ${ }^{20}$ to cause relative shift of the monolayerlike and bilayerlike bands in $A B A$-stacked TLG. Thus, in order to capture some of the finer details of the low-energy spectrum, we include a remote coupling term $t_{2}$ between sites of sublattices $A_{1}$ and $A_{3}$ and $t_{3}$ between $B_{1}$ and $B_{3}$. The Hamiltonian is given as

$$
\mathcal{H}=\left(\begin{array}{cccccc}
U_{1}+U_{0} & v_{F} \pi^{\dagger} & t_{1} & 0 & t_{2} & 0 \\
v_{F} \pi & U_{1} & 0 & 0 & 0 & t_{3} \\
t_{1} & 0 & U_{2}+U_{0} & v_{F} \pi & t_{1} & 0 \\
0 & 0 & v_{F} \pi^{\dagger} & U_{2} & 0 & 0 \\
t_{2} & 0 & t_{1} & 0 & U_{3}+U_{0} & v_{F} \pi^{\dagger} \\
0 & t_{3} & 0 & 0 & v_{F} \pi & U_{3}
\end{array}\right),
$$

where $\pi=p_{x}+i p_{y}$, with $p_{x, y}$ being the components of the in-plane momentum; $U_{1,2,3}$ is the potential in each layer, respectively, $U_{0}$ is the on-site energy at sublattices $A_{1}, B_{2}$, and $A_{3}$, and we defined the eigenstates as $\Psi=$ $\left[\psi_{A 1}, \quad i \psi_{B 1}, \quad \psi_{B 2}, \quad i \psi_{A 2}, \quad \psi_{A 3}, \quad i \psi_{B 3}\right]^{T}$. In the presence of a uniform magnetic field in the $z$ direction, with the gauge $\vec{A}=(0, B x, 0)$ and, for a given sublattice $L, \psi_{L}(y)=$ $\phi_{L} e^{i k_{y} y}$, one obtains the following system of equations:

$$
\begin{gathered}
\mathcal{A}^{+} \phi_{B 1}+t_{1}^{\prime} \phi_{B 2}+t_{2}^{\prime} \phi_{A 3}=\left(\epsilon-u_{1}-u_{0}\right) \phi_{A 1}, \\
\mathcal{A}^{-} \phi_{A 1}+t_{3}^{\prime} \phi_{B 3}=-\left(\epsilon-u_{1}\right) \phi_{B 1}, \\
\mathcal{A}^{-} \phi_{A 2}+t_{1}^{\prime} \phi_{A 1}+t_{1}^{\prime} \phi_{A 3}=\left(\epsilon-u_{2}-u_{0}\right) \phi_{B 2}, \\
\mathcal{A}^{+} \phi_{B 2}=-\left(\epsilon-u_{2}\right) \phi_{A 2}, \\
\mathcal{A}^{+} \phi_{B 3}+t_{1}^{\prime} \phi_{B 2}+t_{2}^{\prime} \phi_{A 1}=\left(\epsilon-u_{3}-u_{0}\right) \phi_{A 3}, \\
\mathcal{A}^{-} \phi_{A 3}+t_{3}^{\prime} \phi_{B 1}=-\left(\epsilon-u_{3}\right) \phi_{B 3},
\end{gathered}
$$

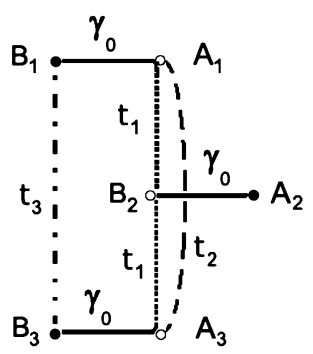

(a) $A B A$

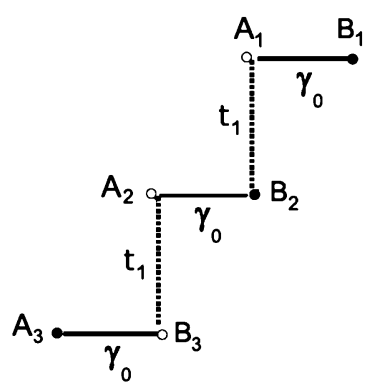

(b) $A B C$
FIG. 1. Diagrammatic scheme of couplings in graphene trilayers for $A B C$ (a) and $A B A$ (b) stackings. where $\epsilon=E / \hbar v_{F}, u_{i}=U_{i} / \hbar v_{F}, t_{i}^{\prime}=t_{i} / \hbar v_{F}$, and $\beta=$ $e B / \hbar v_{F}$ where we defined the operators

$$
\mathcal{A}^{ \pm}=\frac{d}{d x} \pm\left(k_{y}-\beta x\right),
$$

which obey the commutation relation $\left[\mathcal{A}^{+}, \mathcal{A}^{-}\right]=2 \beta$.

For $U_{1}=U_{2}=U_{3}=U$ the system can be easily solved by making use of its reflection symmetry. Thus we can define symmetric and antisymmetric combinations of the spinor components. For the antisymmetric case we obtain $\phi_{G} \equiv \frac{1}{\sqrt{2}}\left(\phi_{A 1}-\phi_{A 3}\right)$, and $\phi_{H} \equiv \frac{1}{\sqrt{2}}\left(\phi_{B 1}-\phi_{B 3}\right)$. That leads to the following pair of coupled equations:

$$
\begin{gathered}
\mathcal{A}^{-} \phi_{G}=-\left(\epsilon-u-t_{3}^{\prime}\right) \phi_{H}, \\
\mathcal{A}^{+} \phi_{H}=\left(\epsilon-u+t_{2}^{\prime}-u_{0}\right) \phi_{G} .
\end{gathered}
$$

For the sake of convenience, let us now define the operator

$$
Z \equiv \mathcal{A}^{-} \mathcal{A}^{+}=\frac{d^{2}}{d x^{2}}-\left(k_{y}-\beta x\right)^{2}-\beta .
$$

We can now decouple the equations to obtain

$$
(Z+2 \beta) \phi_{G}=-\left[\left(\epsilon-u^{\prime}\right)^{2}-(\delta u)^{2}\right] \phi_{G},
$$

which corresponds to the equation that gives the spectrum for a single graphene layer under an effective electrostatic potential $U^{\prime}=U+\left(t_{3}-t_{2}+U_{0}\right) / 2$ as well as a finite gap term given by $\delta U=\left(U_{0}-t_{2}-t_{3}\right) / 2$. Thus the Landau-level spectrum in this case is given by $\epsilon=u^{\prime} \pm \sqrt{2 \beta n+(\delta u)^{2}}$. From Ref. 28 we have $t_{2}=0.04 \mathrm{eV}, t_{3}=-0.02 \mathrm{eV}$, and $U_{0}=0.05 \mathrm{eV}$. Hence the additional effective potential leads to an energy shift of $-5.0 \mathrm{meV}$, and $\delta U=15 \mathrm{meV}$. Thus the effect of the remote coupling terms $t_{2}$ and $t_{3}$, as well as the on-site energy term $U_{0}$, is only to introduce a small shift of the dispersion branches and to generate a small gap in the energy spectrum.

For the symmetric case we have $\phi_{C} \equiv \frac{1}{\sqrt{2}}\left(\phi_{A 1}+\phi_{A 3}\right)$ and $\phi_{D} \equiv \frac{1}{\sqrt{2}}\left(\phi_{B 1}+\phi_{B 3}\right)$. The equations become

and

$$
\begin{gathered}
\mathcal{A}^{+} \phi_{D}+\sqrt{2} t_{1}^{\prime} \phi_{B 2}=\left(\epsilon-u-t_{2}^{\prime}-u_{0}\right) \phi_{C}, \\
\mathcal{A}^{-} \phi_{C}=-\left(\epsilon-u+t_{3}^{\prime}\right) \phi_{D},
\end{gathered}
$$

$$
\begin{gathered}
\mathcal{A}^{-} \phi_{A 2}+\sqrt{2} t_{1}^{\prime} \phi_{C}=\left(\epsilon-u-u_{0}\right) \phi_{B 2}, \\
\mathcal{A}^{+} \phi_{B 2}=-(\epsilon-u) \phi_{A 2},
\end{gathered}
$$

These equations can be decoupled, resulting in the fourth-order differential equation

$$
\left\{Z^{2}+\lambda_{1} Z-\lambda_{2}\right\} \phi_{C}=0,
$$

where $\quad \lambda_{1}=\left(\epsilon-u-u_{0}\right)(\epsilon-u)+\left(\epsilon-u+t_{3}^{\prime}\right)(\epsilon-u-$ $\left.u_{0}-t_{2}^{\prime}\right)+2 \beta$, and $\lambda_{2}=-\left(\epsilon-u-u_{0}\right)(\epsilon-u)\left(\epsilon-u+t_{3}^{\prime}\right)$ $\left(\epsilon-u-u_{0}-t_{2}^{\prime}\right)+2\left(t_{1}^{\prime}\right)^{2}(\epsilon-u)\left(\epsilon-u+t_{3}^{\prime}\right)$. This equation is similar to the one describing bilayer graphene. A second-order equation can be obtained by calculating the roots of the second-order equation as

$$
\left\{Z-z_{+}\right\}\left\{Z-z_{-}\right\} \phi_{C}=0,
$$

with

$$
z_{ \pm}=-\frac{\lambda_{1}}{2} \pm \sqrt{\left(\frac{\lambda_{1}}{2}\right)^{2}+\lambda_{2}}
$$


where we set $k_{y}=0$, since this term only introduces a shift of the wave function. In particular, for $u_{0}=t_{2,3}=0$ the equations yield results that are identical to those of a gapless singlelayer and bilayer graphene, i.e., the Landau levels are found as the solutions of $2 \beta(n+1)=z_{ \pm}$. As in the previous case, the addition of remote coupling terms introduces a gap in the spectrum.

A more realistic description of TLG structures should take into account asymmetries between the different layers, which can be brought about by the interaction with a substrate or by gating. Previous numerical studies ${ }^{21}$ have indicated that a layer asymmetry can lift the valley degeneracy of the TLG Landaulevel spectrum by breaking the inversion symmetry of the system. In order to obtain analytical solutions that incorporate the effect of layer symmetry breaking in the spectrum, let us now consider the case $U_{1} \neq U_{2} \neq U_{3}$. In addition, we now consider $t_{2}=t_{3}=U_{0}=0$, since we assume that the shifts caused by the layer potentials are more significant than the effect of these terms. A simple substitution allows us to write

$$
\begin{aligned}
& {\left[\mathcal{A}^{+} \mathcal{A}^{-}+\left(\epsilon-u_{1}\right)^{2}\right] \phi_{A 1}=t_{1}^{\prime}\left(\epsilon-u_{1}\right) \phi_{B 2},} \\
& {\left[\mathcal{A}^{+} \mathcal{A}^{-}+\left(\epsilon-u_{3}\right)^{2}\right] \phi_{A 3}=t_{1}^{\prime}\left(\epsilon-u_{3}\right) \phi_{B 2} .}
\end{aligned}
$$

In addition, we also have

$$
\left[\mathcal{A}^{-} \mathcal{A}^{+}+\left(\epsilon-u_{2}\right)^{2}\right] \phi_{B 2}=t_{1}^{\prime}\left(\epsilon-u_{2}\right)\left(\phi_{A 1}+\phi_{A 3}\right) .
$$

As in the previous case, we introduce symmetric and antisymmetric combinations of wave functions, and let us also define $\Delta=\left(u_{1}-u_{3}\right) / 2, s=\left(u_{1}+u_{3}\right) / 2$, and $\delta_{j}=\epsilon-u_{j}$, $j=1,2,3$, in order to simplify the notation. After some algebra we obtain the following sixth-order differential equation for $\phi_{B 2}$ :

$$
\begin{aligned}
& \left\{\left[\mathcal{A}^{-} \mathcal{A}^{+}+\delta_{1}^{2}+2 \beta\right]\left[\mathcal{A}^{-} \mathcal{A}^{+}+\delta_{2}^{2}\right]\left[\mathcal{A}^{-} \mathcal{A}^{+}+\delta_{3}^{2}+2 \beta\right]\right. \\
& \quad-t_{1}^{\prime 2} \delta_{2}(\epsilon-s)\left[\mathcal{A}^{-} \mathcal{A}^{+}+\delta_{1}^{2}+2 \beta\right]-t_{1}^{\prime 2} \delta_{2}(\epsilon-s) \\
& \left.\quad \times\left[\mathcal{A}^{-} \mathcal{A}^{+}+\delta_{3}{ }^{2}+2 \beta\right]+4 t_{1}^{\prime 2} \Delta^{2} \delta_{2}(\epsilon-s)\right\} \phi_{B 2}=0 .
\end{aligned}
$$

It is seen that for $U_{1}=U_{2}=U_{3}$ (i.e., $\delta_{1}=\delta_{2}=\delta_{3}, \Delta=0$ ), we recover the previous solutions. One can rewrite Eq. (22) as

$$
\left[Z^{3}+\alpha_{1} Z^{2}+\alpha_{2} Z+\alpha_{3}\right] \phi_{B 2}=0
$$

with the $Z$ operator defined above and

$$
\begin{aligned}
\alpha_{1} \equiv & \delta_{1}^{2}+\delta_{2}^{2}+\delta_{3}^{2}+4 \beta \\
\alpha_{2} \equiv & \left(\delta_{1}^{2}+2 \beta\right)\left(\delta_{3}^{2}+2 \beta\right)+\left(\delta_{1}^{2}+2 \beta\right) \delta_{2}^{2}+\left(\delta_{3}^{2}+2 \beta\right) \delta_{2}^{2} \\
& -t_{1}^{\prime 2} \delta_{2}\left(\delta_{1}+\delta_{3}\right), \\
\alpha_{3} \equiv & \left(\delta_{1}^{2}+2 \beta\right) \delta_{2}^{2}\left(\delta_{3}^{2}+2 \beta\right)-2 \beta t_{1}^{\prime 2} \delta_{2}\left(\delta_{1}+\delta_{3}\right) \\
& -t_{1}^{\prime 2} \delta_{1} \delta_{2} \delta_{3}\left(\delta_{1}+\delta_{3}\right) .
\end{aligned}
$$

This equation can be written as

$$
\left\{Z-Z_{1}\right\}\left\{Z-Z_{2}\right\}\left\{Z-Z_{3}\right\} \phi_{B 2}=0,
$$

where $Z_{j}, j=1,2,3$ are the three roots of the cubic equation, Eq. (14). Therefore the spinor component $\phi_{B 2}$ is a solution of

$$
-\frac{d^{2} \phi_{B 2}}{d x^{2}}+\left(k_{y}-\beta x\right)^{2} \phi_{B 2}=-\left(Z_{j}+\beta\right) \phi_{B 2} .
$$

For zero magnetic field, this equation allows us to obtain planewave solutions for each dispersion branch. The dispersion relation can be obtained by setting $Z_{j}=-k^{2}$. It can be immediately seen that the energy gap at $k=0$ can be found by solving the equation $\alpha_{3}=0$. For finite magnetic fields, the solutions are expressed in terms of Hermite polynomials. Therefore for the Landau levels we obtain the relation $Z_{j}=$ $-2 \beta(n+1)$. Thus the energies are found by solving the algebraic equation

$$
-[2 \beta(n+1)]^{3}+\alpha_{1}[2 \beta(n+1)]^{2}-\alpha_{2}[2 \beta(n+1)]+\alpha_{3}=0 .
$$

It is evident that for $U_{1}=U_{2}=U_{3}$, we have $\Delta=0$ and the last term of Eq. (13) vanishes. The spectrum should then consist of a superposition of the spectra of single-layer graphene and bilayer graphene, as shown in previous work. ${ }^{20}$

\section{III. $A B C$ STACKING}

Let us consider three coupled graphene layers in the $A B C$ stacking configuration. For the sake of simplicity, let us retain only the nearest-neighbor coupling terms. In this case, the Hamiltonian can be written as

$$
\mathcal{H}=\left(\begin{array}{cccccc}
U_{1} & v_{F} \pi^{\dagger} & t & 0 & 0 & 0 \\
v_{F} \pi & U_{1} & 0 & 0 & 0 & 0 \\
t & 0 & U_{2} & v_{F} \pi & 0 & 0 \\
0 & 0 & v_{F} \pi^{\dagger} & U_{2} & 0 & t \\
0 & 0 & 0 & 0 & U_{3} & v_{F} \pi^{\dagger} \\
0 & 0 & 0 & t & v_{F} \pi & U_{3}
\end{array}\right),
$$

where $U_{1,2,3}$ is the potential in each layer, respectively, and we defined the eigenstates as before.

We obtain the following system of equations:

$$
\begin{gathered}
\mathcal{A}^{+} \phi_{B 1}+t^{\prime} \phi_{B 2}=\left(\epsilon-u_{1}\right) \phi_{A 1}, \\
\mathcal{A}^{-} \phi_{A 1}=-\left(\epsilon-u_{1}\right) \phi_{B 1}, \\
\mathcal{A}^{-} \phi_{A 2}+t^{\prime} \phi_{A 1}=\left(\epsilon-u_{2}\right) \phi_{B 2}, \\
\mathcal{A}^{+} \phi_{B 2}-t^{\prime} \phi_{B 3}=-\left(\epsilon-u_{2}\right) \phi_{A 2}, \\
\mathcal{A}^{+} \phi_{B 3}=\left(\epsilon-u_{3}\right) \phi_{A 3}, \\
\mathcal{A}^{-} \phi_{A 3}-t^{\prime} \phi_{A 2}=-\left(\epsilon-u_{3}\right) \phi_{B 3},
\end{gathered}
$$

where $\epsilon=E / \hbar v_{F}, \quad u_{i}=U_{i} / \hbar v_{F}, \quad t^{\prime}=t / \hbar v_{F}$, and $\beta=$ $e B / \hbar v_{F}$. In order to decouple these equations, let us first obtain $\phi_{B 1}$ and $\phi_{A 3}$ in terms of $\phi_{A 1}$ and $\phi_{B 3}$ from the second and fifth equations as

$\phi_{B 1}=-\frac{1}{\left(\epsilon-u_{1}\right)} \mathcal{A}^{-} \phi_{A 1}, \quad \phi_{A 3}=\frac{1}{\left(\epsilon-u_{3}\right)} \mathcal{A}^{+} \phi_{B 3}$,

and substitute these expressions in the first and sixth equations, respectively, to give

$$
\begin{aligned}
& \mathcal{A}^{+} \mathcal{A}^{-} \phi_{A 1}-t^{\prime}\left(\epsilon-u_{1}\right) \phi_{B 2}=-\left(\epsilon-u_{1}\right)^{2} \phi_{A 1}, \\
& \mathcal{A}^{-} \mathcal{A}^{+} \phi_{B 3}-t^{\prime}\left(\epsilon-u_{3}\right) \phi_{A 2}=-\left(\epsilon-u_{3}\right)^{2} \phi_{B 3} .
\end{aligned}
$$

Equations (22a) and (22b) allow us to obtain $\phi_{B 2}$ and $\phi_{A 2}$ in terms of $\phi_{A 1}$ and $\phi_{B 3}$, respectively. Thus by substituting them in Eqs. (27) and (28), respectively, and after some tedious algebra, one can obtain a sixth-order differential 
equation as

$$
\begin{aligned}
& \left\{\left[\mathcal{A}^{-} \mathcal{A}^{+}+\delta_{1}^{2}+2 \beta\right]\left[\mathcal{A}^{-} \mathcal{A}^{+}+\delta_{2}^{2}\right]\left[\mathcal{A}^{-} \mathcal{A}^{+}+\delta_{3}^{2}-2 \beta\right]\right. \\
& \quad-t^{\prime 2} \delta_{2} \delta_{3}\left[\mathcal{A}^{-} \mathcal{A}^{+}+\delta_{1}^{2}+2 \beta\right] \\
& \left.\quad-t^{\prime 2} \delta_{1} \delta_{2}\left[\mathcal{A}^{-} \mathcal{A}^{+}+\delta_{3}^{2}-2 \beta\right]+t^{\prime 4} \delta_{1} \delta_{3}\right\} \phi_{A 1}=0 .
\end{aligned}
$$

It is interesting to compare Eqs. (13) and (23). The former remains invariant if one switches the potentials in layers 1 and 3. Equation (23), on the other hand, is found to be invariant under an interchange of potentials between the topmost and lowest layers together with a reversal of the magnetic field. This reflects the different symmetries of each stacking of TLG.

As before, we can obtain the Landau-level spectrum by rewriting Eq. (23) as

$$
\left[Z^{3}+\gamma_{1} Z^{2}+\gamma_{2} Z+\gamma_{3}\right] \phi_{A 1}=0
$$

with $Z \equiv \mathcal{A}^{-} \mathcal{A}^{+}$, and

$$
\begin{aligned}
\gamma_{1} \equiv & \delta_{1}^{2}+\delta_{2}^{2}+\delta_{3}^{2}, \\
\gamma_{2} \equiv & \left(\delta_{1}^{2}+2 \beta\right)\left(\delta_{3}^{2}-2 \beta\right)+\left(\delta_{1}^{2}+2 \beta\right) \delta_{2}^{2}+\left(\delta_{3}^{2}-2 \beta\right) \delta_{2}^{2} \\
& -\left(t^{\prime}\right)^{2}\left(\delta_{1}+\delta_{3}\right) \delta_{2}, \\
\gamma_{3} \equiv & -\left(t^{\prime}\right)^{2} \delta_{2}\left[\delta_{3}\left(\delta_{1}^{2}+2 \beta\right)+\delta_{1}\left(\delta_{3}^{2}-2 \beta\right)\right]+\left(t^{\prime}\right)^{4} \delta_{1} \delta_{3} \\
& +\left(\delta_{1} \delta_{2} \delta_{3}\right)^{2}+2 \beta\left(\delta_{3}^{2}-\delta_{1}^{2}\right) \delta_{2}^{2}-4\left(\beta \delta_{2}\right)^{2} .
\end{aligned}
$$

This equation can be written as

$$
\left\{Z-Z_{1}\right\}\left\{Z-Z_{2}\right\}\left\{Z-Z_{3}\right\} \phi_{A 1}=0,
$$

where $Z_{j}, j=1,2,3$ are the three roots of the cubic equation, Eq. (24). Therefore the spinor component $\phi_{A 1}$ is found as a solution of

$$
-\frac{d^{2} \phi_{A 1}}{d x^{2}}+\left(k_{y}-\beta x\right)^{2} \phi_{A 1}=-\left(Z_{j}+\beta\right) \phi_{A 1} .
$$

For the particular case of $U_{1}=U_{2}=U_{3}=0$ and zero magnetic field, we obtain plane-wave solutions by setting $Z_{j}=-k^{2}$, where $k$ is the in-plane wave vector. Thus Eq. (24) can be rewritten as

$$
\epsilon^{6}-\left(3 k^{2}+2 t^{\prime 2}\right) \epsilon^{4}+\left(3 k^{4}-2 k^{2} t^{\prime 2}+t^{\prime 4}\right) \epsilon^{2}-k^{6}=0 .
$$

Let us now consider the low-energy limit $\epsilon \ll t^{\prime}$. That allows us to neglect the higher-order powers of $\epsilon$ to obtain

$$
\epsilon \approx \frac{k^{3}}{t^{\prime 2}} \frac{1}{\sqrt{1-2 k^{2} / t^{\prime 2}+3 k^{4} / t^{\prime 4}}} .
$$

Thus, for small wave vectors, the dispersion relation increases with the third power of $k$.

The Landau levels can be obtained using the relation $Z_{j}=$ $-2 \beta(n+1)$, which leads to the algebraic equation

$$
-[2 \beta(n+1)]^{3}+\gamma_{1}[2 \beta(n+1)]^{2}-\gamma_{2}[2 \beta(n+1)]+\gamma_{3}=0 .
$$

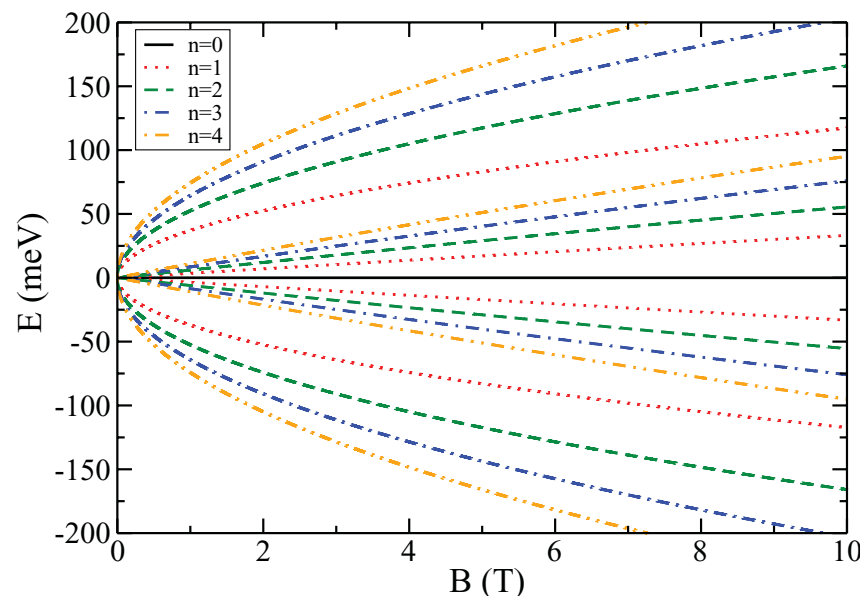

FIG. 2. (Color online) The lowest Landau levels as function of magnetic field for $A B A$-stacked graphene trilayers calculated from Eq. (18), with $U_{1}=U_{2}=U_{3}=0$, for $n=0$ (black solid lines), $n=1$ (red dotted lines), $n=2$ (green dashed lines), $n=3$ (blue dot-dashed lines), and $n=4$ (yellow dot-dot-dashed lines).

As seen above, for zero potential in each layer, we can find a simpler algebraic relation for the energy, namely

$$
\begin{aligned}
\epsilon^{6} & -\left[6 \beta(n+1)+2 t^{\prime 2}\right] \epsilon^{4} \\
+ & {\left[12 \beta^{2}(n+1)^{2}-4 \beta(n+1) t^{\prime 2}+t^{\prime 4}-4 \beta^{2}\right] \epsilon^{2} } \\
& -8 \beta^{3}(n+1)^{3}+8 \beta^{3}(n+1)=0 .
\end{aligned}
$$

For $\epsilon \ll t$ we can then obtain

$$
\epsilon \approx \pm \frac{(2 \beta)^{3 / 2}}{t^{\prime 2}} \sqrt{n(n+1)(n+2)} F(\beta, n),
$$

where

$$
F(\beta, n)=\left[1-4 \frac{\beta}{t^{\prime 2}}(n+1)-4 \frac{\beta^{2}}{t^{\prime 4}}+12 \frac{\beta^{2}}{t^{\prime 4}}(n+1)^{2}\right]^{-1 / 2} \text {. }
$$

For small fields (i.e., $\left.\beta \ll t^{\prime 2}\right), F(\beta, n) \approx 1$. Therefore, in the limit of low energies and small fields, the Landau levels should approximately depend on the magnetic field as $B^{3 / 2}$, in agreement with the results of Ref. 13.

\section{NUMERICAL RESULTS}

Let us first consider the $A B A$ case. Figure 2 shows the field dependence of the low-lying Landau levels calculated from Eq. (18), with $U_{1}=U_{2}=U_{3}=0$, for $n=0$ (black solid lines), $n=1$ (red dotted lines), $n=2$ (green dashed lines), $n=$ 3 (blue dot-dashed lines), and $n=4$ (yellow dot-dot-dashed lines). We find that for every value of $n$ there are two different types of low-energy branches: (1) those that depend linearly on the magnetic field (i.e., bilayerlike behavior), and (2) branches that display a $B^{1 / 2}$ dependence (monolayerlike branches). A third set of bilayerlike branches with linear dependencies on $B$ are found around $E= \pm \sqrt{2} t_{1}$, not shown in the figure.

The effect of a potential difference between the layers on the energy spectrum as a function of magnetic field is shown in Fig. 3. As in the previous case, the figure shows 


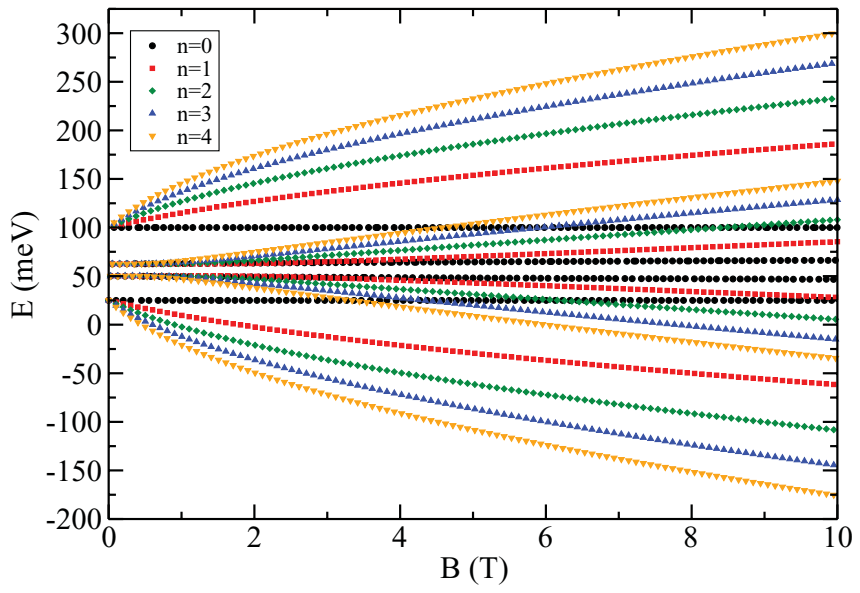

FIG. 3. (Color online) Energy spectrum as function of magnetic field for $A B A$-stacked graphene trilayers, for $U_{1}=100 \mathrm{meV}, U_{2}=$ $50 \mathrm{meV}$, and $U_{3}=25 \mathrm{meV}, n=0$ (black dots), $n=1$ (red squares), $n=2$ (green diamonds), $n=3$ (blue up triangles), and $n=4$ (yellow down triangles).

branches corresponding to $n=0-4$. The potentials in the different graphene layers are $U_{1}=100 \mathrm{meV}, U_{2}=50 \mathrm{meV}$, and $U_{3}=25 \mathrm{meV}$. As seen from Eq. (18), for $B \rightarrow 0$ we have solutions corresponding to $E=U_{j}, j=1,2,3$, and $E=$ $\left(U_{1}+U_{3}\right) / 2$. Thus we find that the monolayerlike branches are shifted creating a gap with magnitude $U_{1}-U_{3}$, whereas for the bilayerlike states a smaller gap opens with magnitude $\left(U_{1}+U_{3}\right) / 2-U_{2}$. One consequence of this difference is the appearance of several level crossings around $E=U_{2}$ and $E=$ $\left(U_{1}+U_{3}\right) / 2-U_{2}$ for small $B$, together with a nonmonotonic dependence of the energy on the magnetic field that evolves to a linear dependence as the magnetic field is increased.

Figure 4 shows the low-energy Landau levels as a function of the potential in the inner layer, for the $A B A$ case, for $n=1$ (red squares), 2 (green up triangles), and 3 (blue squares) for $B=3 \mathrm{~T}, U_{1}=U_{3}=50 \mathrm{meV}$. Notice that (1) the lowest energy levels depend linearly on $U_{2}$ for small $U_{2}\left(\ll U_{1}=\right.$

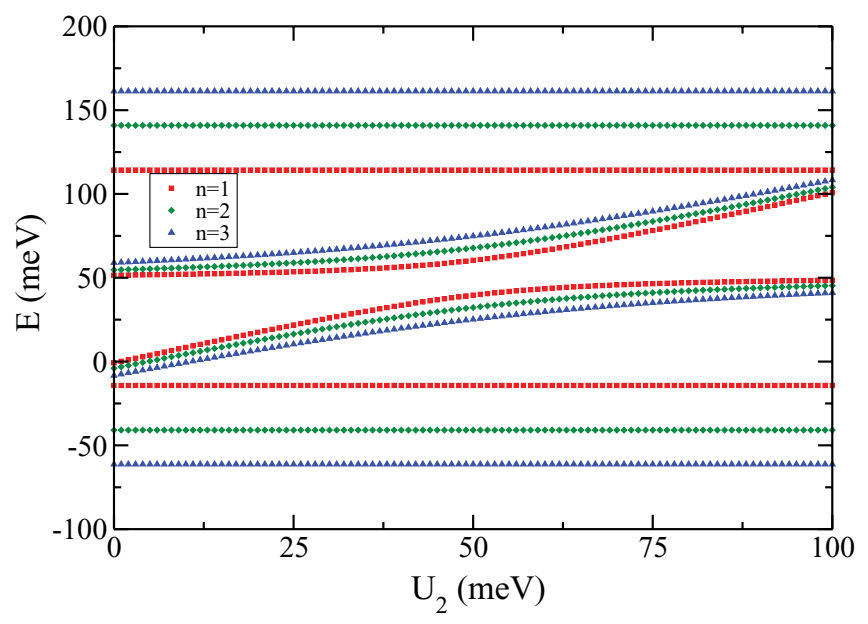

FIG. 4. (Color online) Low-lying Landau levels as function of the potential in the inner layer for $A B A$-stacked graphene trilayers, for $n=1$ (red squares), 2 (green diamonds), and 3 (blue up triangles) for $B=3 \mathrm{~T}, U_{1}=U_{3}=50 \mathrm{meV}$.

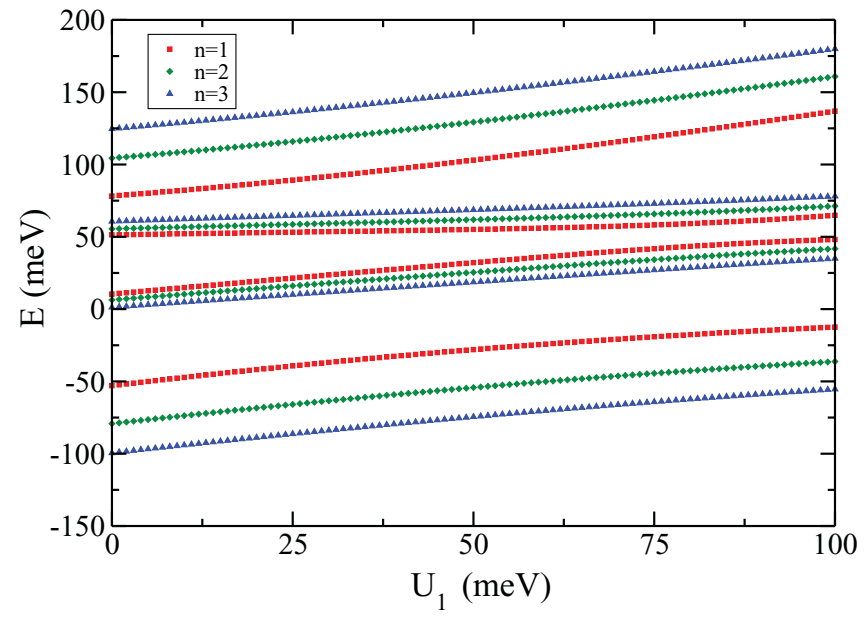

FIG. 5. (Color online) Low-lying Landau levels as function of the potential in the uppermost layer for $A B A$-stacked graphene trilayers, for $n=1$ (red squares), 2 (green diamonds), and 3 (blue up triangles) for $B=3 \mathrm{~T}, U_{2}=50 \mathrm{meV}, U_{3}=25 \mathrm{meV}$.

$\left.U_{3}\right)$ and for large $U_{2}\left(\gg U_{1}=U_{3}\right)$ values, and exhibit an anticrossing behavior for $U_{2} \approx U_{1}=U_{3} ; 2$ ) the higher energy states are very weakly affected by the bias. A different behavior is observed when one varies the potential at the uppermost layer $\left(U_{1}\right)$, as seen in Fig. 5. In contrast with the previous case, the bias is seen to cause a significant shift also on the higher energy Landau levels.

The energy spectrum for the $A B C$ case is shown in Fig. 6(a), as a function of magnetic field and with $U_{1}=U_{2}=U_{3}=0$, for $n=0-4$. For small $B$ values we see a doubly degenerate branch with $E=0$, and a $B^{3 / 2}$ behavior for the remaining
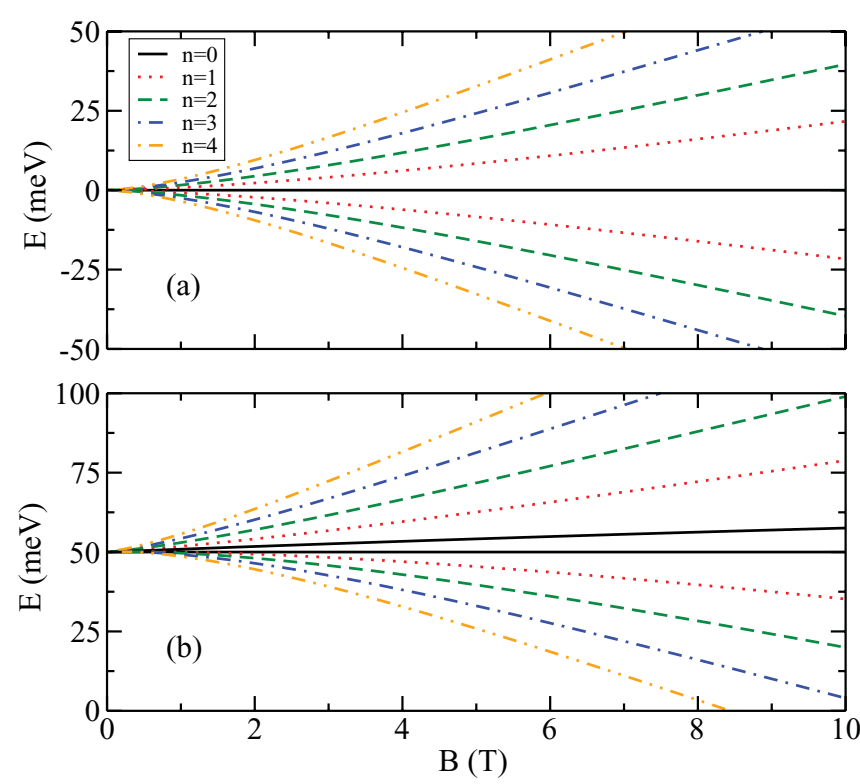

FIG. 6. (Color online) Landau-level spectrum for the trilayer graphene for the $A B C$ stacking, as function of magnetic field, with $U_{1}=U_{2}=U_{3}=0$ (a), and $U_{1}=U_{3}=50 \mathrm{meV}, U_{2}=100 \mathrm{meV}$ (b) for $n=0$ (black solid lines), $n=1$ (red dotted lines), $n=2$ (green dashed lines), $n=3$ (blue dot-dashed lines), and $n=4$ (yellow dot-dot-dashed lines). 


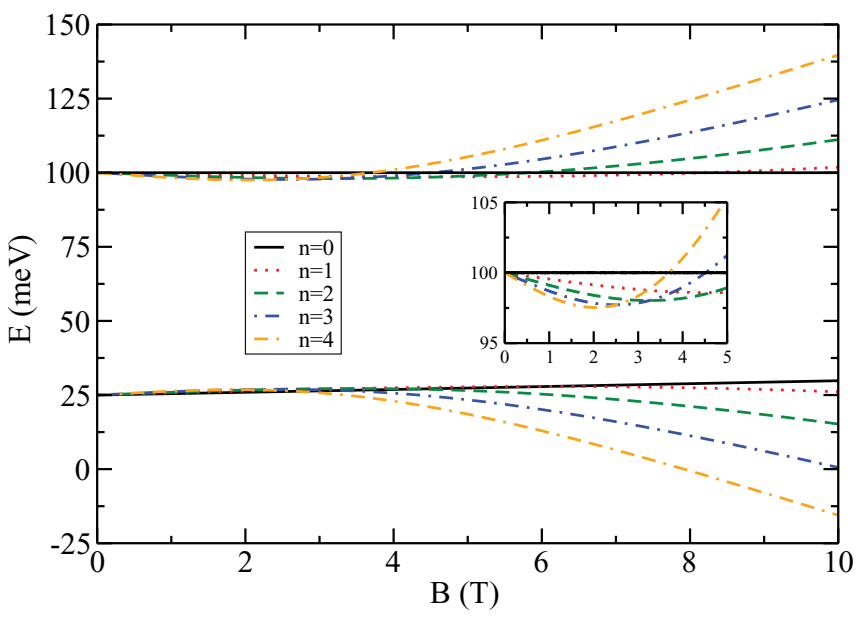

FIG. 7. (Color online) Landau-level spectrum for the trilayer graphene for the $A B C$ stacking, as function of magnetic field, with $U_{1}=100 \mathrm{meV}, U_{2}=50 \mathrm{meV}$, and $U_{3}=25 \mathrm{meV}$, for $n=0$ (black solid lines), $n=1$ (red dotted lines), $n=2$ (green dashed lines), $n=3$ (blue dot-dashed lines), and $n=4$ (yellow dot-dot-dashed lines).

branches, which turns into a linear behavior at large $E$. In comparison with the previous case, the results in the $A B C$ case show the presence of pairs of branches at low energies, whereas in the $A B A$ case one finds two sets of energy levels for each Landau index. That is caused by the fact that, in the $A B C$ case, the remaining four branches are found around $E= \pm t$, with $t \approx 400 \mathrm{meV}$.

Figure 6(b) shows results for an $A B C$ TLG with $U_{1}=$ $U_{3}=50 \mathrm{meV}$, whereas $U_{2}=100 \mathrm{meV}$. In this case, the main effect of the potential difference is the lifting of the degeneracy of the $n=0$ state and a shift of the whole spectrum to lower energy with increasing magnetic field.

In contrast, Fig. 7 shows the LL spectrum for $U_{1}=100$, $U_{2}=50$, and $U_{3}=25 \mathrm{meV}$. The inset shows an enlargement

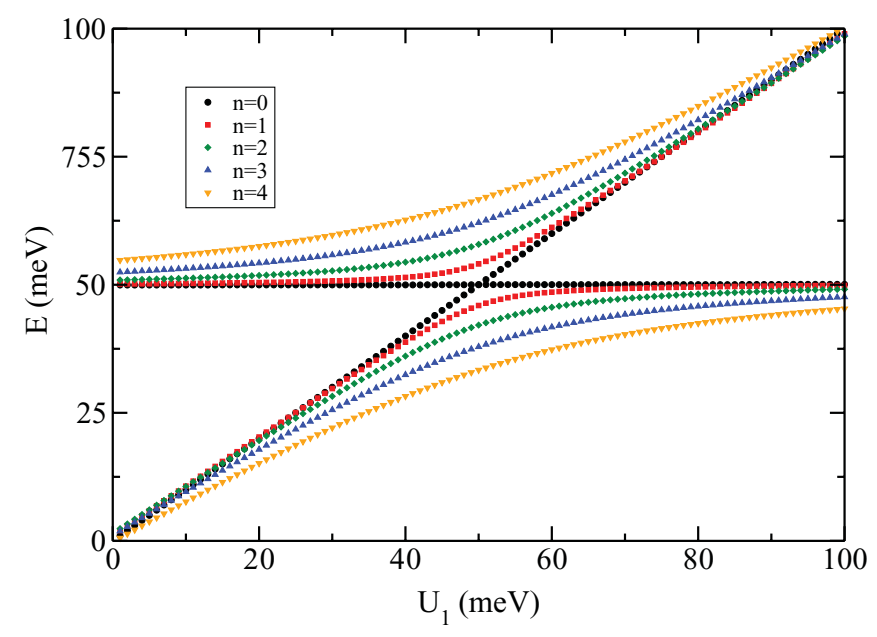

FIG. 8. (Color online) Landau-level spectrum for $A B C$-stacked trilayer graphene as function of $U_{1}$, for $B=3 \mathrm{~T}$ and $U_{2}=U_{3}=$ $50 \mathrm{meV}$, with $n=0$ (black dots), $n=1$ (red squares), $n=2$ (green diamonds), $n=3$ (blue up triangles), and $n=4$ (yellow down triangles).

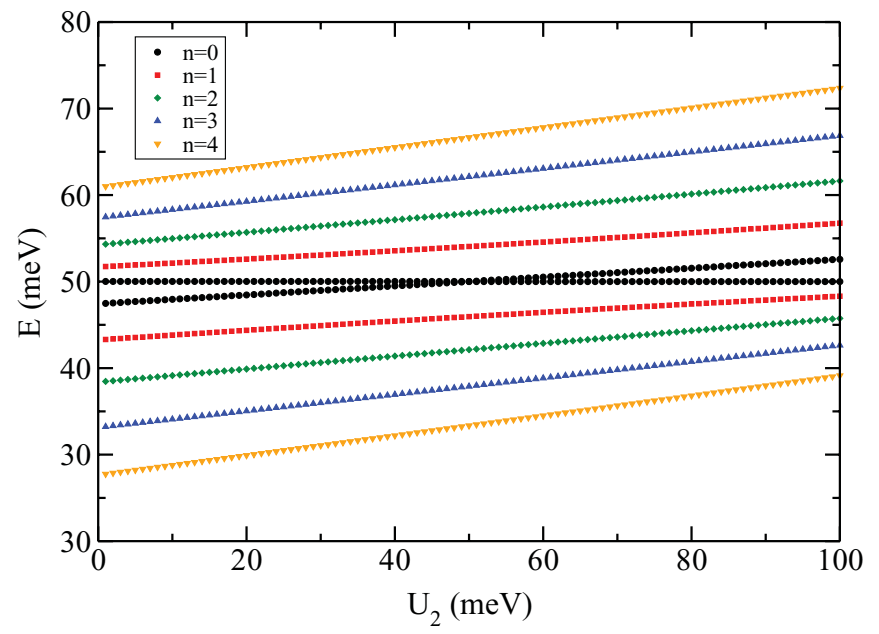

FIG. 9. (Color online) Landau level spectrum for ABC-stacked trilayer graphene as function of $U_{2}$, for $B=3 \mathrm{~T}$ and $U_{1}=U_{3}=$ $50 \mathrm{meV}$, with $n=0$ (black dots), $n=1$ (red squares), $n=2$ (green diamonds), $n=3$ (blue up triangles), and $n=4$ (yellow down triangles).

of the region around $E=100 \mathrm{meV}$. In this case, the bias creates an energy gap, which can be found by setting $\beta=0$ in Eq. (30), which leads to solutions with $E=U_{1}$ and $E=U_{2}$. Notice also the existence of level crossings, as well as the peculiar small magnetic-field behavior where there is a reversal of the ordering of the Landau levels as compared to the regular high magnetic-field behavior.

Results for the dependence of the energy spectrum on $U_{1}$ is shown in Fig. 8, for $B=3 \mathrm{~T}$ and $U_{2}=U_{3}=50 \mathrm{meV}$. As seen, the degeneracy of the $n=0$ is lifted for $U_{1} \neq$ $U_{2}, U_{3}$. Moreover, when the magnitude of the potential in the uppermost layer is increased, the Landau levels tend to become degenerate. A quite distinct picture emerges if one varies instead the potential in the middle layer $\left(U_{2}\right)$, as shown in Fig. 9, for $B=3 \mathrm{~T}$ and $U_{1}=U_{3}=50 \mathrm{meV}$. In contrast with the previous results, the spectrum shows a linear dependence on the potential and there are no degeneracies for the different Landau indices. As in the previous figure, a single Landau level at $E=50 \mathrm{meV}$ is found to be unaffected by the bias.

\section{CONCLUSIONS}

In summary, we obtained exact analytical expressions for the Landau-level spectra of trilayer graphene, within a model that took into account the layer asymmetry induced by different electrostatic potentials in each layer. The expressions were obtained for both the Bernal $(A B A)$ and rhombohedral $(A B C)$ stackings, which were found to display quite distinct behaviors. As shown in previous work, ${ }^{20}$ the Landau-level spectrum for the $A B A$ case in the absence of electrostatic bias between the layers shows both a monolayerlike as well as bilayerlike character, indicated by the different magnetic-field dependence of the spectrum. The addition of a potential difference between the layers shifts the spectrum and creates a tunable gap between the electron and hole states, the size of this energy gap being different for the monolayer and the bilayer energy levels. Level crossings between the monolayer 
and bilayer Landau levels are found for certain values of the magnetic field.

For the $A B C$ case, the Landau levels have a magneticfield dependence which, in the absence of bias, has a $B^{3 / 2}$ dependence for low energies. ${ }^{13}$ For stronger magnetic fields the Landau levels exhibit a linear $B$ dependence. The introduction of electrostatic bias in the system lifts the degeneracy of the $n=0$ levels and creates a tunable gap. The results show also the existence of level crossings at small magnetic fields. This model can be refined by taking into account second-nearestneighbor terms, as well as remote coupling between the lowest and uppermost layers.

In this paper, we considered a model Hamiltonian that neglects second-nearest-neighbor couplings in each layer, as well as between adjacent layers. Future work may consider the effect of these additional terms on the spectrum, in order to give a more precise account of the electron and hole behaviors in trilayer graphene. In particular, the trigonal warping induced by the additional terms has been shown to lead to important effects, such as the splitting of quantum Hall plateaus caused by a Lifshitz transition induced by trigonal warping. ${ }^{23}$ Moreover, a significant modification of the field dependence of the low-energy Landau levels may arise as a consequence of long-range electron-electron interactions. Such effect has been already shown to be relevant in the case of bilayer graphene. ${ }^{30}$ A systematic study of the effects of such interactions, however, goes beyond the scope of the present paper.

Note added. As we were preparing this manuscript for publication, we became aware of a similar calculation of Landau levels in trilayer graphene, by Yuan et al. ${ }^{31}$

\section{ACKNOWLEDGMENTS}

This work was supported by the Brazilian Council for Research (CNPq), the National Council for the Improvement of Higher Education (CAPES), the Flemish Science Foundation (FWO-Vl), the Belgian Science Policy (IAP), and the bilateral projects between Flanders and Brazil and the $\mathrm{CNPq}$ and FWO-V1.
${ }^{1}$ A. H. Castro Neto, F. Guinea, N. M. R. Peres, K. S. Novoselov, and A. Geim, Rev. Mod. Phys. 81, 109 (2009).

${ }^{2}$ M. I. Katsnelson, K. S. Novoselov, and A. K. Geim, Nat. Phys. 2, 620 (2006).

${ }^{3}$ J. M. Pereira Jr., F. M. Peeters, A. Chaves, and G. A. Farias, Semicond. Sci. Technol. 25, 033002 (2010).

${ }^{4}$ J. Cserti and G. Dávid, Phys. Rev. B 74, 172305 (2006).

${ }^{5}$ G. Dávid and J. Cserti, Phys. Rev. B 81, 121417(R) (2010).

${ }^{6}$ J. M. Pereira Jr., P. Vasilopoulos, and F. M. Peeters, Appl. Phys. Lett. 90, 132122 (2007).

${ }^{7}$ E. McCann and V. I. Fal'ko, Phys. Rev. Lett. 96, 086805 (2006).

${ }^{8}$ E. V. Castro, K. S. Novoselov, S. V. Morozov, N. M. R. Peres, J. M. B. Lopes dos Santos, Johan Nilsson, F. Guinea, A. K. Geim, and A. H. Castro Neto, Phys. Rev. Lett. 99, 216802 (2007).

${ }^{9}$ H. Min and A. H. MacDonald, Phys. Rev. B 77, 155416 (2008).

${ }^{10}$ B. Partoens and F. M. Peeters, Phys. Rev. B 74, 075404 (2006).

${ }^{11}$ A. A. Avetisyan, B. Partoens, and F. M. Peeters, Phys. Rev. B 81, 115432 (2010).

${ }^{12}$ M. Aoki and H. Amawashi, Solid State Commun. 142, 123 (2007).

${ }^{13}$ F. Guinea, A. H. Castro Neto, and N. M. R. Peres, Phys. Rev. B 73, 245426 (2006).

${ }^{14}$ S. Yuan, H. De Raedt, and M. I. Katsnelson, Phys. Rev. B 82, 235409 (2010).

${ }^{15}$ H. Min, P. Jain, S. Adam, and M. D. Stiles, Phys. Rev. B 83, 195117 (2011).

${ }^{16}$ T. Taychatanapat, K. Watanabe, T. Taniguchi, and P. Jarillo-Herrero, Nat. Phys. (to be published).

${ }^{17}$ L. Zhang, Y. Zhang, J. Camacho, M. Khodas, and I. A. Zaliznyak, e-print arXiv:1103.6023 (to be published).
${ }^{18}$ A. Kumar, W. Escoffier, J. M. Poumirol, C. Faugeras, D. P. Arovas, M. M. Fogler, F. Guinea, S. Roche, M. Goiran, and B. Raquet, e-print arXiv:1104.1020 (to be published).

${ }^{19}$ M. Koshino and E. McCann, Phys. Rev. B 80, 165409 (2009).

${ }^{20}$ M. Koshino and E. McCann, Phys. Rev. B 83, 165443 (2011).

${ }^{21}$ M. Koshino and E. McCann, Phys. Rev. B 81, 115315 (2010).

${ }^{22}$ W. Bao, Z. Zhao, H. Zhang, G. Liu, P. Kratz, L. Jing, J. Velasco, Jr., D. Smirnov, and C. N. Lau, Phys. Rev. Lett. 105, 246601 (2010).

${ }^{23}$ W. Bao, L. Jing, Y. Lee, J. Velasco Jr., P. Kratz, D. Tran, B. Standley, M. Aykol, S. B. Cronin, D. Smirnov, M. Koshino, E. McCann, M. Bockrath, and C. N. Lau, e-print arXiv:1103.6088 (to be published).

${ }^{24}$ Y. Liu, S. Goolaup, C. Murapaka, W. S. Lew, and S. K. Wong, ACS Nano. 4, 7087 (2010).

${ }^{25}$ S. H. Jhang, M. F. Craciun, S. Schmidmeier, S. Tokumitsu, S. Russo, M. Yamamoto, Y. Skourski, J. Wosnitza, S. Tarucha, J. Eroms, and C. Strunk, e-print arXiv:1106.4995 (to be published).

${ }^{26}$ C. H. Lui, Z. Li, Z. Chen, P. V. Klimov, L. E. Brus, and T. F. Heinz, Nano Lett. 11, 164 (2011).

${ }^{27}$ A. A. Avetisyan, B. Partoens, and F. M. Peeters, Phys. Rev. B 80, 195401 (2009).

${ }^{28}$ M. Koshino and E. McCann, Phys. Rev. B 79, 125443 (2009).

${ }^{29}$ C. H. Lui, Z. Li, K. F. Mak, E. Cappelluti, and T. F. Heinz, e-print arXiv:1105.4658 (to be published).

${ }^{30}$ D. S. L. Abergel and T. Chakraborty, Phys. Rev. Lett. 102, 056807 (2009).

${ }^{31}$ S. Yuan, R. Roldán, and M. I. Katsnelson, Phys. Rev. B 84, 125455 (2011). 